\title{
Marker-Less Stage Drift Correction in Super-Resolution Microscopy Using the Single-Cluster PHD Filter
}

\author{
Isabel Schlangen, José Franco, Jérémie Houssineau, William T. E. Pitkeathly, \\ Daniel Clark, Ihor Smal, and Colin Rickman
}

\begin{abstract}
Fluorescence microscopy is a technique which allows the imaging of cellular and intracellular dynamics through the activation of fluorescent molecules attached to them. It is a very important technique because it can be used to analyze the behavior of intracellular processes in vivo in contrast to methods like electron microscopy. There are several challenges related to the extraction of meaningful information from images acquired from optical microscopes due to the low contrast between objects and background and the fact that point-like objects are observed as blurred spots due to the diffraction limit of the optical system. Another consideration is that for the study of intracellular dynamics, multiple particles must be tracked at the same time, which is a challenging task due to problems such as the presence of false positives and missed detections in the acquired data. Additionally, the objective of the microscope is not completely static with respect to the cover slip due to mechanical vibrations or thermal expansions which introduces bias in the measurements. In this paper, a Bayesian approach is used to simultaneously track the locations of objects with different motion behaviors and the stage drift using image data obtained from fluorescence microscopy experiments. Namely, detections are extracted from the acquired frames using image processing techniques, and then these detections are used to accurately estimate the particle positions and simultaneously correct the drift introduced by the motion of the sample stage. A single cluster Probability Hypothesis Density (PHD) filter with object classification is used for the estimation of the multiple target state assuming different motion behaviors. The detection and tracking methods are tested and their performance is evaluated on both simulated and real data.
\end{abstract}

Manuscript received March 15, 2015; revised July 20, 2015; accepted November 13, 2015. Date of publication December 07, 2015; date of current version January 21, 2016. The work of I. Schlangen was supported by the Edinburgh Super-Resolution Imaging Consortium under Grant MR/K01563X/1. The work of C. Rickman was supported in part by the Edinburgh Super-Resolution Imaging Consortium under Grant MR/K01563X/1, in part by the Medical Research Council under Grant MR/K018639/1, and in part by the European Research Council under Grant 111507. The work of D. Clark was supported by EPSRC under Grant EP/J015180/1. The work of I. Smal was supported by VENI Grant \#639.021.128 from the Netherlands Organization for Scientific Research (NWO). The guest editor coordinating the review of this manuscript and approving it for publication was Prof. Jean-Christophe Olivo-Marin.

I. Schlangen, J. Franco, J. Houssineau, W. T. E. Pitkeathly, D. Clark, and C. Rickman are with the School of Engineering and Physical Sciences, Heriot-Watt University, Edinburgh EH14 4AS, U.K. (e-mail: is117@hw.ac.uk; jf139@hw. ac.uk; j.houssineau@hw.ac.uk; e.w.pitkeathly@hw.ac.uk; d.e.clark@hw.ac.uk; c.rickman@hw.ac.uk).

I. Smal is with the Biomedical Imaging Group Rotterdam, Departments of Medical Informatics and Radiology, Erasmus MC, 3015 GE Rotterdam, The Netherlands (e-mail: i.smal@erasmusmc.nl).

This paper has supplemental downloadable multimedia material available at http://ieeexplore.ieee.org, provided by the authors. The Supplementary Materials contain two MP4 files showing estimations of the sensor motion as described in the paper. This material is $6.9 \mathrm{MB}$ in size.

Color versions of one or more of the figures in this paper are available online at http://ieeexplore.ieee.org.

Digital Object Identifier 10.1109/JSTSP.2015.2506402
Index Terms-Biomedical imaging, molecular imaging, microscopy, particle tracking, probability density function, estimation, simultaneous localization and mapping, filtering.

\section{INTRODUCTION}

$\mathbf{T}$ HE study of intracellular processes is essential in many biomedical areas such as cancer research, drug discovery, and many fields of biology. For instance, understanding the machinery of cellular secretion of neurotransmitters is very important in the study of diseases such as diabetes, Alzheimer's and Parkinson's. Motion is an essential aspect of these processes, and yet the acquisition of data to analyse movement within cells is far from being a trivial task. The size of the particles of interest is an important obstacle - optical microscopy, for instance, is limited by the diffraction barrier, while other techniques that can resolve smaller objects, such as electron microscopy are lethal to living cells, ruling out their use as tools to analyse their dynamics.

Fluorescence microscopy is a particularly interesting method for the study of intracellular behaviour. This technique is a type of optical microscopy where the imaged light is emitted from fluorescent molecules, called fluorophores, that are attached to the objects of interest. Fluorophores can be genetically coded to attach to particular kinds of protein so that specific types of objects are analysed at individual times [1]. This way, very small particles can be observed through the light emitted by the fluorophores while excluding uninteresting objects from the analysis.

The ability to tag protein molecules with fluorophores and observe them through a microscope has been extensively used by biologists to develop their understanding about protein behaviour in several ways. Super-resolution techniques such as (fluorescent) Photo-Activated Localisation Microscopy (PALM/fPALM) [2], [3] allow the imaging of very large amounts of molecules through successive activation-acquisition-localisation cycles. Some methods focus on determining properties of protein types from their global behaviour in the cell, such as Fluorescence Recovery After Photobleaching (FRAP) or Fluorescence Loss In Photobleaching (FLIP) [4]. In this article, the focus will be on tracking individual protein molecules through time from live cell PALM data acquired with Total Internal Reflection Fluorescence (TIRF) microscopy. This imaging paradigm utilises a special mode of sample illumination, known as an evanescent field, to exclusively excite 
fluorescent molecules within $\sim 200 \mathrm{~nm}$ from the imaging surface which the cell membranes are adhered to [5]. By limiting the excitation volume, no fluorescence is caused deeper inside the cell; therefore, the images are not contaminated by light from out-of-focus planes. In the past, approaches such as single particle tracking PALM (sptPALM) [6] which combine PALM and single particle tracking have been used to extract statistical information about the motion of groups of particles.

Although sub-cellular dynamics can be observed using fluorescence microscopy, excessive light exposure causes damage to the fluorophores (photobleaching). This process releases free oxygen radicals and causes phototoxicity within live cells [1]. To reduce this harm, the excitation light used to activate the fluorophores must be limited, which results in a decrease in the signal-to-noise ratio of the acquired data. Additionally, since the observed particles are smaller than the resolution of the microscope, they appear as the microscope's point spread function and a localisation step must be carried out in order to determine their physical position from the images. These two problems can be addressed systematically using digital image processing techniques, and several different approaches have been utilised in the past to do so [7], [8].

When imaging dynamic events on the nano-scale, every measure must be taken to prevent movement of the microscope objective lens with respect to the sample. Microscopes are typically mounted onto air-cushioned optics benches which greatly reduce the effects of vibrations from external sources. Small lateral shifts can sometimes occur whilst using on-line refocusing optomechanics during the time lapse imaging. However, an often unavoidable cause of stage drift is due to small temperature variations over the whole metal microscope body. Live cell samples often have to be kept at $\sim 37^{\circ} \mathrm{C}$. Microscopes are fitted with warm-air sample incubators to locally maintain the temperature around the sample stage. Still, thermal equilibrium of the whole microscope is difficult to maintain, thus small expansions and contractions of the microscope body cause the sample to move with respect to the objective lens.

Several post-acquisition techniques have been proposed to correct this stage drift, either by using fiducial markers in the sample (e.g., [9]) or marker-less methods like template matching [10]. The use of external markers is often undesirable: their brightness can be very different to the molecules of interest, thus non-optimal imaging settings are used to prevent camera pixel saturation. Furthermore, their position with respect to the cell cannot be predetermined and therefore are often situated in undesirable areas. Statistical methods like in [11] are performed separately from the tracking procedure on the whole image. Template matching, on the other hand, relies on distinct image features like a consistent background structure which is not always available. On small time scales below $10 \mathrm{~s}$ the drift manifests itself as relatively linear and gradual shifts in the sample position over several time frames, sometimes with short pauses followed by a change in direction. On larger time scales the drift will often appear random in motion.

In this paper, the sensor drift is estimated in parallel to the estimation of the intracellular object trajectories using stochastic methods, based only on the detections that are extracted from the image sequence. The usage of stochastic estimation is preferable since uncertainty in the image acquisition and in the detec- tion of presumed objects is taken into account. Furthermore, the presented bias estimation technique can be performed without the presence of fiducial image features, using only the measurements obtained by the microscope images to estimate this motion alongside with the motion of the objects of interest based on a probabilistic scheme. The performance of the proposed method is assessed in the following by comparing the estimation results on two subsets of the same data, one containing static beads and one that only shows actual intracellular activity.

Manual frame-to-frame particle tracking is prohibitively time consuming due to high particle numbers and large data sets and it is prone to human error [12], [13]. Due to this, automated tracking methods are essential in the analysis of complex sub-cellular mechanisms with a large number of components. The extension from single-target to multiple-target tracking introduces problems such as the presence of false positives and missed detections which must be handled appropriately [14].

In multi-object target tracking, one usually differentiates between deterministic and probabilistic approaches [15]. Deterministic methods typically comprise an intensity-based localisation and a heuristic data association step, such as sptPALM where a naive tracking strategy is used to associate measurements to tracks according to their spatial proximity. Other methods are graph-theoretical linking and optical flow track assignment [12], heuristics such as greedy approaches, assignment based on the Hungarian algorithm and more [13]. Although they are usually time-efficient, a major drawback of these approaches is that they do not cope well with difficult conditions such as occlusions, complex backgrounds, a low signal-to-noise ratio (SNR) or overlapping and high density of particle trajectories. Probabilistic methods, on the other hand, involve Bayesian filtering that gives a prediction about the future target state and updates the predicted belief once a measurement is associated to it. This technique has the advantage that it considers probabilistic uncertainty based on the reliability of the sensor which can compensate for spurious or incomplete data.

Various multiple target tracking techniques are generalised from the single target tracking case by introducing a heuristic that assigns measurements to single-target tracking filters, such as the Joint Probabilistic Data Association Filter (JPDAF, [16], [17]) or the Multiple Hypothesis Tracker (MHT, [14], [18]). A recently proposed class of filters uses a Bayesian approach which eliminates the necessity of data association in favour of propagating a multitarget distribution directly, such as the Probability Hypothesis Density (PHD) filter [19], [20]. This is advantageous since in many cases data association can be computationally expensive and unreliable. Still, there are several track extraction techniques for PHD filters which can be used if the individual trajectories are of interest, see e.g., [21] or [22]. A comparison between the cardinalised PHD (CPHD) and the JPDA approaches can be found in [23]. Recent derivations have yielded filters that estimate the joint state of conditional processes [24], which are particularly suitable for problems where the state of the sensor changes with time, such as sensor calibration [25], Simultaneous Localisation And Mapping (SLAM) [26], or camera calibration [27]. This approach will be used to correct the position of the microscope while simultaneously estimating the location of the individual protein molecules. A sim- 
ilar method was introduced in [28] which combines the sensor motion estimation with the Hypothesised filter for Independent Stochastic Populations (HISP). A PHD filter approach has been presented before in [29]; since the images can contain objects with different motion models, a PHD filter with classification is introduced in the following to distinguish groups of objects with different behaviours and to make use of the classification for the sensor calibration.

The rest of the article is divided as follows: In Section II, the methodology to extract measurements from microscopy images is described and the sensor calibration based on PHD filtering with object classification is discussed. Section III shows the results of applying this methodology on synthetic and real data. Finally, conclusions are presented in Section IV.

\section{Methodology}

The proposed method is comprised of three parts which are detailed in this section. First, measurements are extracted from the images provided by the microscope (Section II-A). Then, these are supplied to a filter that jointly estimates both the locations of the detected particles (Section II-B) and the stage drift (Section II-C).

\section{A. Particle Detection}

The first step in the estimation process consists of extracting the coordinates of the protein molecules from each frame of the acquired video sequence. This process strongly depends on the nature of the data that is used. In photo-activated localisation microscopy (PALM) which we will focus on in this paper (see e.g., [30]), the fluorescence appears in the images as circular spots on a dark and noisy background, their shape resulting from the point spread function specific to the microscope [31]. The point spread function is best modelled as an Airy disk, but a Gaussian approximation is accurate and simple to compute [32].

Automatic particle detection techniques for fluorescence microscopy usually involve a noise reduction step, followed by signal enhancement and finally signal thresholding to extract the measurements [31]. Noise reduction is usually necessary due to the low SNR of the images, while the signal enhancement step may highlight pixels in the image likely to contain particles while de-emphasizing those caused by background noise, and then a decision is made on which pixels correspond to particles during the thresholding step. Below, a detection method based on wavelets will be described in more detail, its performance is discussed in Section III.

In the following, let us denote the greyscale input image by

$$
\mathcal{I}=\{I(i, j)\}_{i \in\{1 \ldots m\}, j \in\{1 \ldots n\}} \in \mathbb{R}^{m \times n} .
$$

$I(i, j)$ being the intensity of the pixel at position $(i, j)$.

To obtain measurements from the video sequence, image denoising and enhancement is performed using the Isotropic Undecimated Wavelet Transform (IUWT) as described in [31], which is suitable for numerous applications in astronomy and biology. The idea is to exploit the band-pass properties of the wavelet transform by decomposing the image into $K$ wavelet planes that contain different frequency levels to suppress unwanted structures such as high-frequency noise or low-frequency background.

In particular, let us define the one-dimensional kernel $H=[1 / 16,1 / 4,3 / 8,1 / 4,1 / 16]$. By sequential row- and

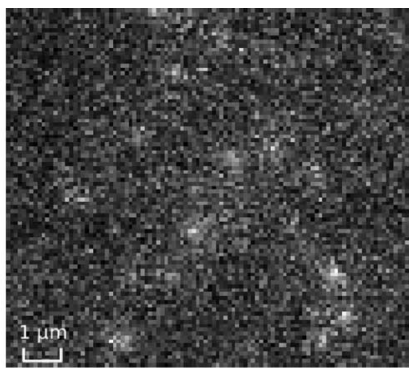

(a)

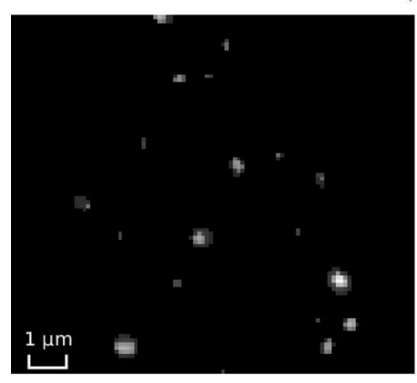

(b)

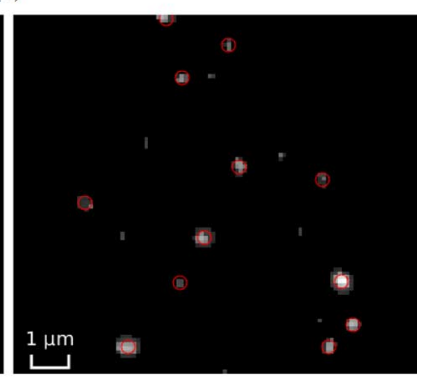

(c)
Fig. 1. Particle detection steps on a detail view of a frame of live cell PALM data. (a) Original image (b) À trous wavelet denoising (c) Detections.

column-wise convolution of the input image $\mathcal{I}$ with $H$, a sequence $\left\{\mathcal{I}_{k}\right\}_{k \in\{1 \ldots K\}}$ is obtained where $K$ denotes the number of applied convolution operations. Calculating the differences $\mathcal{W}_{k}=\mathcal{I}_{k-1}-\mathcal{I}_{k}$ where $W_{k}(i, j)=I_{k-1}(i, j)-I_{k}(i, j)$ for $i \in\{1 \ldots m\}$ and $j \in\{1 \ldots n\}$, we find the à trous wavelet decomposition:

$$
\mathcal{I}=\mathcal{I}_{K}+\sum_{k=1}^{K} \mathcal{W}_{k} .
$$

Each of the layers $\mathcal{W}_{k}$ contains features of different sizes, so the image noise can easily be filtered out by thresholding each of the layers before reconstructing the image via (2), replacing $\mathcal{W}_{k}$ by the thresholded images.

Lastly, isolated disjoint clusters of more than two non-zero pixels are considered as detections. The position of each detection is defined as the centre of mass within each cluster. The complete process is illustrated in Fig. 1.

\section{B. Multiple Target Tracking}

Having extracted the locations of the protein molecules using the acquired microscope images, it is of interest to analyse their spatio-temporal dynamics using target tracking.

Probabilistic tracking is the process of obtaining an estimate of the state of the system through time based on the received measurements, while minimising the effects of noise and possibly obtaining information on variables that are not directly observable such as the velocity of each object [14]. Many tracking techniques also give information on the likelihood of the state estimate, which allows a more in-depth analysis. Filtering techniques are particularly interesting because they use the models of how the system evolves through time and how observations are generated from it to give statistically sound estimates of the state. This problem has been widely studied for single target tracking, but the generalisation to multiple targets is problematic due to the appearance of false positives and missed detections 
as well as intersections of object trajectories as mentioned earlier. In fluorescence microscopy, the objects of interest are often just above the noise level which makes them hardly perceivable, i.e., missed detections can be caused by temporally inactive fluorophores or because the detector fails to distinguish the object from the background noise. In turn, false positives can be caused by autofluorescence of the background or because noisy image regions lead to misclassification.

As it was previously mentioned, approaches such as Multiple Hypothesis Tracking deal with these problems through the use of heuristics to assign individual measurements to a collection of single target filters based on data association [14]. Recent work has generalised single target Bayes filtering to the multiple target case by using Random Finite Sets (RFS) to represent a collection of objects [20]. A RFS contains a random number of vectors which are in turn random, so this parametrisation is well suited to multiple target filtering. In this framework, the multi-target Bayes filter offers a theoretical solution to the optimal filtering problem, but as its single target counterpart it is intractable in terms of its implementation and requires certain approximations in order to be used in practical estimation applications.

A widely used approximation that makes the problem tractable is the PHD filter [19]. This filter propagates the first moment $D(\mathbf{x})$ of the multi-target posterior, known as the PHD or intensity, which is a function defined on the single-target state space which indicates the expected number of targets in any of its regions. If the multi-target probability distribution is denoted $p_{k}(\mathbf{X})$, then the following equality holds:

$$
\int_{S} D(\mathbf{x}) \mathrm{d} \mathbf{x}=\int|\mathbf{X} \cap S| p_{k}(\mathbf{X}) \delta \mathbf{X}=N_{k}(S),
$$

where $N_{k}(S)$ denotes the expected number of targets in set $S$ and $|S|$ is the cardinality of $S$. The integral of the form $\int \ldots \delta \mathbf{X}$ is a set integral [19]. The process and measurement models used by the PHD filter are based on the following assumptions:

1) From time step $k$ to time step $k+1$, each target $\mathbf{x}_{k}$ survives with probability $P_{S}\left(\mathbf{x}_{k}\right)$ evolving into $\mathbf{x}_{k+1}=f_{k}\left(\mathbf{x}_{k}\right)$, or it disappears with probability $1-P_{S}\left(\mathbf{x}_{k}\right)$.

2) New targets may appear at each time step according to an independent birth process.

3) A target with state $\mathbf{x}$ produces a measurement $\mathbf{z}=\zeta_{k}(\mathbf{x})$ with probability $P_{D}(\mathbf{x})$ or is not detected with probability $1-P_{D}(\mathbf{x})$.

4) False alarms are produced at each time step according to a given clutter distribution.

These models offer great flexibility when representing the system of interest. In particular, for fluorophore tracking, the motion model $f_{k}$ can be specified according to the expected behaviour of the object of interest- constant position for fixed features in the field of view, Brownian motion for particles that exhibit short range diffusion, or constant velocity for more motile particles on random or pre-defined pathways. The measurement model $\zeta_{k}$, on the other hand, may simply be a scaling that transforms points in the metric coordinates of the stage into pixel coordinates in the image plane.

As an alternative to [29] where the classical PHD filter is used, the proposed method will involve $N_{\mathrm{mm}}$ different motion models to account for multiple co-existing behaviours and also make use of this information for the sensor track estimation. In the following, the index $i$ will indicate the relation of a variable to one particular motion model. A similar approach was used as well in [26] which propagates the sum of two Poisson processes as a single process with different motion models. The approach of Rezatofighi et al. [33] also makes use of multiple Markov models, alongside with transition probabilities to model behavioural changes, however this approach does not provide classification. A derivation of the original PHD filter with a single motion model can be found in [19].

The validity of the proposed approach can be justified as follows. Assuming that $N_{\mathrm{mm}}=2$ and denoting $X_{k}^{i}$ the RFS corresponding to objects with motion model $i \in\{1,2\}$, we consider the following prediction-update scheme:

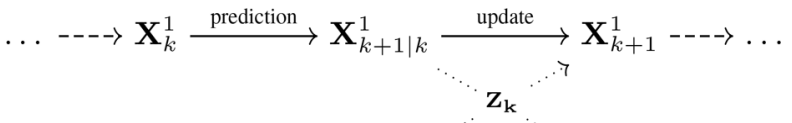

$$
\begin{aligned}
& \ldots \mathbf{X}_{k}^{2} \stackrel{\text { prediction }}{\longrightarrow} \mathbf{X}_{k+1 \mid k}^{2} \stackrel{\text { update }}{\longrightarrow} \mathbf{X}_{k+1}^{2} \stackrel{\lrcorner}{--->} \ldots
\end{aligned}
$$

The prediction on $\mathbf{X}_{k}^{1}$ and $\mathbf{X}_{k}^{2}$ is performed separately, but since the set $Z_{k}$ contains the observations originating from both $\mathbf{X}_{k}^{1}$ and $\mathbf{X}_{k}^{2}$, the update step has to be performed jointly. In practice, when updating the intensity of the process $\mathbf{X}_{k}^{1}$ with the observation set $\mathbf{Z}_{k}$, the process $\mathbf{X}_{k}^{2}$ is viewed as a source of false positives along with the true clutter. Since only the marginal intensities of $\mathbf{X}_{k+1}^{1}$ and $\mathbf{X}_{k+1}^{2}$ are computed, these processes have to be assumed independent in order to iterate the above-described prediction-update scheme. Furthermore, the birth process splits into two independent birth processes that create objects for each motion model. Finally, the updates can be joined again to a single PHD by summing the two separate PHDs due to the independence assumption.

All these considerations are synthesised in the following RFS process and measurement models:

$$
\begin{gathered}
\mathbf{X}_{k}^{i}=\boldsymbol{B}_{k} \cup \bigcup_{\substack{\mathbf{x} \in \mathbf{X}_{k-1}^{i} \\
\mathbf{Z}_{k}}} \boldsymbol{Y}_{k \mid k-1}^{i}(\mathbf{x}), \\
\bigcup_{\substack{\mathbf{x} \in \mathbf{X}_{k}^{i} \\
1 \leq i \leq N_{\mathrm{mm}}}} \boldsymbol{T}_{k}(\mathbf{x}),
\end{gathered}
$$

where $\boldsymbol{B}_{k}$ and $\mathbf{K}_{k}$ are the birth and clutter random finite sets, respectively, and

$$
\begin{aligned}
\boldsymbol{Y}_{k \mid k-1}^{i}(\mathbf{x}) & = \begin{cases}\left\{f_{k}^{i}(\mathbf{x})\right\}, & \text { with probability } P_{S}(\mathbf{x}) \\
\emptyset, & \text { with probability } 1-P_{S}(\mathbf{x})\end{cases} \\
\boldsymbol{T}_{k}(\mathbf{x}) & =\left\{\begin{array}{ll}
\left\{\zeta_{k}(\mathbf{x})\right\}, & \text { with probability } P_{D}(\mathbf{x}) \\
\emptyset, & \text { with probability } 1-P_{D}(\mathbf{x})
\end{array} .\right.
\end{aligned}
$$

In these equations, $f_{k}^{i}$ are the $N_{\mathrm{mm}}$ single-target process models and $\zeta_{k}$ is the measurement model. The survival and detection probabilities can in general be state-dependent and time-varying. For the purpose of this work, however, they will be treated as constant so that

$$
P_{S}(\mathbf{x})=P_{S}, \quad P_{D}(\mathbf{x})=P_{D} .
$$

In order to manage appearing targets, an alternative strategy to include new targets in the posterior is used with measurement driven births [34], [35]. Where no a priori information is available on where protein molecules are likely to appear, measure- 
ments are used to determine likely positions for new target locations. Using this strategy, the prediction (9) and update (10) equations of the PHD filter are the following:

$$
\begin{aligned}
& D_{k+1^{\prime} k}^{i}(\mathbf{x})=\int t_{k}^{i}\left(\mathbf{x} \mid \mathbf{x}^{\prime}\right) D_{k}^{i}\left(\mathbf{x}^{\prime}\right) \mathrm{d} \mathbf{x}^{\prime}, \\
& D_{k+1}^{i}(\mathbf{x})=\left(1-P_{D}\right) D_{k+1 \mid k}^{i}(\mathbf{x}) \\
& +\sum_{\mathbf{z} \in \mathbf{Z}_{k}} \frac{P_{D} g_{k}(\mathbf{z} \mid \mathbf{x}) D_{k+1 \mid k}^{i}(\mathbf{x})+w_{\gamma} \gamma_{\mathbf{z}}(\mathbf{x})}{c(\mathbf{z})+\sum_{j=1}^{N_{\operatorname{mm}}} \int g_{k}\left(\mathbf{z} \mid \mathbf{x}^{\prime}\right) D_{k+1 \mid k}^{j}\left(\mathbf{x}^{\prime}\right)+w_{\gamma} \gamma_{\mathbf{z}}\left(\mathbf{x}^{\prime}\right) \mathrm{d} \mathbf{x}^{\prime}},
\end{aligned}
$$

where the Markov transition $t_{k}^{i}\left(\cdot \mid \mathbf{x}^{\prime}\right)$ is the probability density of a target under motion model $i$ conditioned on its previous state being $\mathbf{x}^{\prime}, g_{k}(\mathbf{z} \mid \mathbf{x})$ is the observation likelihood of $\mathbf{z}$ conditioned on single-target state $\mathbf{x}, c(\mathbf{z})$ is the PHD of the clutter process, the function $\gamma_{\mathbf{z}}(\mathbf{x})$ models the state space distribution of a target that produces measurement $\mathbf{z}$ and $w_{\gamma}$ is the weight that is assigned to this new component. $D_{k}^{i}$ is in fact the first moment of the RFS $\mathbf{X}_{k}^{i}$ as defined above. Note that this process involves $N_{\mathrm{mm}}$ PHD filters in parallel, but they interact through the global normalisation in the update (10).

The filter can be implemented in practice by choosing an appropriate form for $D(x)$. The Gaussian Mixture (GM) implementation represents it as a sum of Gaussian components with different means and covariances [36]:

$$
D_{k}^{i}(\mathbf{x})=\sum_{j=0}^{N_{k}} w_{j} \mathcal{N}\left(\mathbf{x} ; \boldsymbol{\mu}_{j}, \boldsymbol{P}_{j}\right) .
$$

After applying (9) or (10), respectively, the predicted and updated PHDs are also Gaussian mixtures due to the linearity of the Bayesian filter equations. At each time step, the state estimate can be extracted by adding up the weights to obtain an estimate $n_{k}$ of the number of targets, and then using the means of the $n_{k}$ Gaussian terms with the highest weights as a state estimate. In addition to this, track labels can be obtained by using the approach described in [37].

\section{Bias Estimation}

Observing objects of molecular size requires great care to make the microscope objective as still as possible with respect to the observed sample. Sometimes, there will still be some motion caused by factors such as thermal expansion or contraction of the sample stage or other sources of perturbation which can introduce a time-varying bias on the acquired measurements. One way of eliminating this bias is by including static markers in the sample from which it can be directly measured, but this may be undesirable due to possible interference with the objects of interest or other considerations. In this article, a stochastic method to estimate the microscope drift is used that evaluates the likelihood of frame-to-frame object propagation which can be used with or without static markers.

This problem can be formulated as the joint estimation of the multitarget state $\mathbf{X}_{k}$ and the sensor state $\mathbf{y}_{k}$ which are respectively the locations of the protein molecules within the sample and the relative position of the microscope objective to the sample based on the measurement history $\mathbf{Z}_{1: k}$. Related problems are SLAM [26], camera calibration [27], or more general parameter calibration [25]; the idea was also utilised in [28] and [29]. According to the general definition, the joint probability distribution can be written as follows:

$$
p\left(\mathbf{X}_{k}, \mathbf{y}_{k} \mid \mathbf{Z}_{1: k}\right)=p\left(\mathbf{X}_{k} \mid \mathbf{Z}_{1: k}, \mathbf{y}_{k}\right) p\left(\mathbf{y}_{k} \mid \mathbf{Z}_{1: k}\right) .
$$

The ensemble of the sensor position and the particle state can be modelled as a single cluster process where the measurements acquired from the particles are conditioned on the position of the sensor. The joint estimation of the sensor state and the multiple target state can be achieved with the single cluster PHD filter [24]. This is a Sequential Monte Carlo approach where the distribution of the sensor is represented as a set of particles, each with an associated multi-motion-model PHD representing in turn the state of the observed objects conditioned on that particular sensor state.

As with all particle based approaches, a choice on the number $N$ of hypothesised biases has to be made. In general, the use of more particles will more accurately represent the approximated distribution, while using less particles will decrease the computational burden of the algorithm. For particle $i$ at time step $k$, the hypothesised state of the microscope will be denoted $\mathbf{y}_{k}^{i}$, the associated PHD $D_{k}^{i, j}$ with motion model $j$ and the weight $w_{k}^{i}$. Additionally, the motion model of the sensor has to be specified, which is the probability distribution $f_{k}\left(\mathbf{y} \mid \mathbf{y}^{\prime}\right)$ of the sensor having state $\mathbf{y}$ at time step $k$ conditioned on it having state $\mathbf{y}^{\prime}$ in the previous time step. For the application that is studied here it suffices to model the motion of the microscope as a Brownian motion process, with a covariance chosen to agree with the expected range of motion of the sensor.

For initialisation, since no information is available, the initial values of all particles are chosen to be the zero vector and every PHD is initialised to zero. The weights of all particles start with the same value of $1 / N$. Afterwards, prediction and update steps are iterated as the data arrives.

Prediction is done by sampling a new sensor state from each particle from the chosen process model $M_{k-1}$ :

$$
\mathbf{y}_{k}^{i} \sim M_{k-1}\left(\mathbf{y} \mid \mathbf{y}_{k-1}^{i}\right)
$$

Having done this, the PHD is also predicted as described above.

When a set of measurements $\mathbf{Z}_{k}$ arrives, each particle's PHD is updated with it using the measurement model conditioned on its hypothesised sensor state. This can be easily done by removing the particle's bias from each measurement and performing a regular PHD update as above. After that, the weights of each particle are updated using the multi-target likelihood:

$$
L\left(\mathbf{Z}_{k} \mid D_{k}^{i, j}, \mathbf{y}_{k}^{i}\right)=\frac{\hat{L}\left(\mathbf{Z}_{k} \mid D_{k}^{i, j}, \mathbf{y}_{k}^{i}\right)}{\int \hat{L}\left(\mathbf{Z}_{k} \mid D_{k}^{i, j}, \mathbf{y}\right) \mathrm{d} \mathbf{y}},
$$

where

$$
\begin{array}{r}
\hat{L}\left(\mathbf{Z}_{k} \mid D_{k}^{i, j}, \mathbf{y}\right)=\exp \left(-\int P_{D} D_{k}^{i, j}(\mathbf{x} \mid \mathbf{y}) \mathrm{d} \mathbf{x}\right) \\
\left.\prod_{\mathbf{z} \in \mathbf{Z}_{k}}\left(c(\mathbf{z})+\int P_{D} g(\mathbf{z} \mid \mathbf{x}, \mathbf{y}) D_{k}^{i, j}(\mathbf{x} \mid \mathbf{y}) \mathrm{d} \mathbf{x}\right)\right) .
\end{array}
$$

One could choose to use all motion models at once or only a selection, e.g., one could consider to use just the static features in the image as it was done in the experiments for this work. 
When computing the new weights, it is unnecessary to compute the denominator of the above expression since the weights will be normalised to sum to one:

$$
\begin{aligned}
\tilde{w}_{k}^{i} & =L\left(\mathbf{Z}_{k} \mid D_{k}^{i}, \mathbf{y}_{k}^{i}\right) \\
w_{k}^{i} & =\frac{\tilde{w}_{k}^{i}}{\sum_{n=1}^{N} \tilde{w}_{k}^{n}} .
\end{aligned}
$$

Particle filters approximate the posterior using a finite number of particles. To increase the accuracy of the representation, it is desirable to have more particles in the more probable regions of the state space. Thus, a resampling step might be carried out if necessary to rearrange the particles. Due to its form, the multiobject likelihood is multimodal and spiky, so resampling must be done carefully to relocate the particles to the correct regions.

In the proposed solution, the effective sample size is checked in every iteration to evaluate the distribution of importance amongst the particles. The effective sample size is obtained from the particle weights via

$$
N_{\mathrm{eff}}=\frac{1}{\sum_{i=1}^{N}\left(w_{k}^{i}\right)^{2}}
$$

as described in [38]. If this value falls below $N / 2$, a roulette resampling step is performed. One could also consider to use more sophisticated methods such as importance sampling with rejection correction (see [38] or [29]).

There are several choices to extract the estimated sensor trajectory from the particle filter, e.g., the mean position could be determined in every time step. In cases of multivariate distributions, however, this method can fail very easily. Therefore, a Maximum-A-Posteriori (MAP) approach was chosen for the following experiments which extracts the particle position with the highest weight per frame. This method leads to less smooth trajectories, but it assures a more reliable performance over time.

\section{Algorithm 1: Algorithm for bias estimation}

Input: Set of particles $\left\{\left(\mathbf{y}_{k}^{i}, D_{k}^{i, j}\right)\right\}_{i=1}^{N}$

Set of measurements $\mathbf{Z}_{k+1}$

Output: Updated set of particles $\left\{\left(\mathbf{y}_{k+1}^{i}, D_{k+1}^{i, j}\right)\right\}_{i=1}^{N}$ Prediction

for $i=1$ to $N$ do

$$
\begin{aligned}
& \text { Sample } \mathbf{y}_{k+1}^{i} \sim \mathcal{N}\left(\mathbf{y}_{k}^{i}, \Sigma_{s}\right) \\
& D_{k+1 \mid k}^{i, j} \leftarrow \operatorname{PHDPrediction}\left(D_{k}^{i, j}\right)
\end{aligned}
$$

end

$$
\begin{aligned}
& \begin{array}{l}
\text { Update } \\
\text { for } i=1 \text { to } N \text { do }
\end{array} \\
& \quad \hat{\mathbf{Z}}_{k+1}^{i} \leftarrow \text { removeBias }\left(\mathbf{Z}_{k+1}, \mathbf{y}_{k+1}^{i}\right) \\
& \text { for } j=1 \text { to } N_{m m} \text { do } \\
& \quad D_{k+1}^{i, j} \leftarrow \operatorname{PHDUpdate}\left(D_{k+1 \mid k}^{i, j}, \hat{\mathbf{Z}}_{k+1}^{i}\right) \\
& \quad \text { end } \\
& \text { end } \\
& \text { if } N_{e f f}<N / 2 \text { then } \\
& \quad \text { resampleBiases }() \\
& \text { end }
\end{aligned}
$$

\section{RESULTS}

This section analyses the performance of the detection methods and the tracking algorithm separately to give more insight in both aspects. Unless stated otherwise, standard deviations stated below are assumed to be the same in both image dimensions.

\section{A. Detections}

Although the PHD framework accounts for the sensor performance through the observation model $\zeta_{k}$ and the detection probability $P_{D}$, a decent detection method is helpful to obtain good tracking results: too many missed detections could lead to the loss of a track, whereas too many false positives increase the complexity unnecessarily and can lead to ambiguities. Therefore, it is useful to evaluate the performance of the detector based on synthetic data where ground truth is available. The method described in [39] was used for the simulation since it mimics all different aspects of TIRF microscopy imaging using realistic point spread function (PSF) models, sensor and photon noise, and z-depth dependent particle intensity variation due to the evanescent field used for excitation. Unlike the works in [39], where deformable particle models were presented, all simulated particles are sub-diffraction limit.

Different backgrounds can strongly influence the visibility of the objects inside, thus two different backgrounds were considered for comparison; both are of size $511 \mathrm{px} \times 401 \mathrm{px}$. As a generic background, white noise was sampled from a Gaussian distribution with mean 0 and standard deviation 150 on a 16 -bit greyscale image ${ }^{1}$ with initial intensity 2000 and then convolved with a Gaussian distribution having a standard deviation of $10 \mathrm{px}$ in the manner described in [31]. The second background was extracted from real data, where the cell membrane shows high autofluorescence with changing intensities. These two backgrounds were superimposed with 100 different sets of around 200 objects with different initial intensities $I_{0} \in\{50,100,200\}$; for details of the simulation method, cf. [39]. One example frame per simulation setting is shown in Fig. 2.

For evaluation, the optimal sub-pattern assignment (OSPA) distance between the detection results and the ground truth was computed for every frame with a cut-off value of $c=10$ and with $p=2$ [40]. In Fig. 3, the results were averaged over all 100 object sets. The table demonstrates that the performance improves significantly for higher contrast between objects and background in terms of distance and cardinality. Furthermore, the detector gives slightly better results on the simulated background; this might result from the uniformity of the latter, whereas the real background shows areas of high intensity where the SNR is locally low.

The real data used for the experiments on stage drift estimation shows good contrast between a uniform background and the targets of interest, similar to the data shown in Fig. 2(e).

\section{B. Drift Correction}

In order to have a realistic scenario for the target tracking, live PALM data was used for all experiments. A sample frame is shown in Fig. 5(a). This dataset was acquired on an Olympus

\footnotetext{
${ }^{1} \mathrm{~A}$ 16-bit greyscale image allows values between 0 and 65,535.
} 


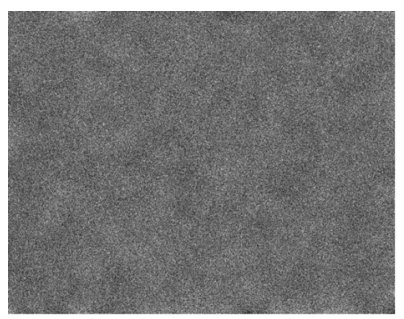

(a)

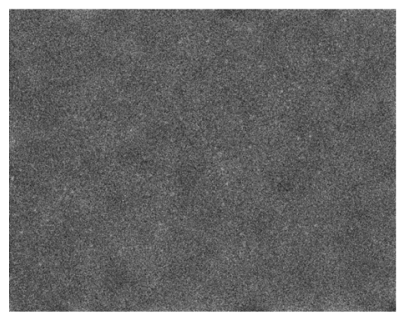

(c)

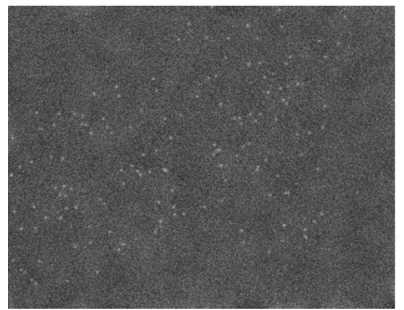

(e)

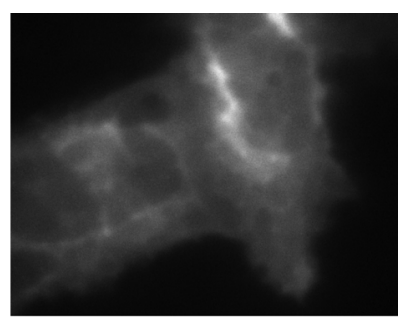

(b)

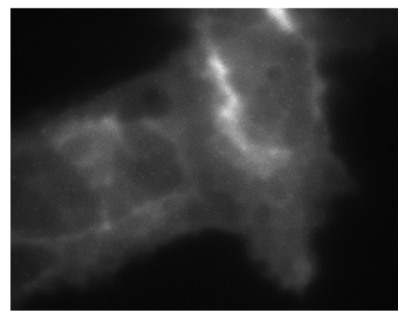

(d)

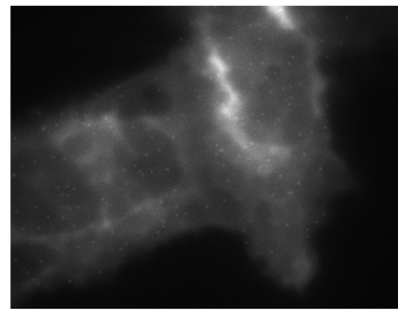

(f)
Fig. 2. Six simulated TIRF images based on the same object locations. The left column shows several object intensities on generic background, the right column shows the same objects on a background which was obtained from real data. (a) Initial intensity: $I_{0}=50$. (b) Initial intensity: $I_{0}=50$. (c) Initial intensity: $I_{0}=100$. (d) Initial intensity: $I_{0}=100$. (e) Initial intensity: $I_{0}=200$. (f) Initial intensity: $I_{0}=200$.

\begin{tabular}{|c|c|c|c|c|c|}
\hline & $I_{0}$ & $\mu_{\text {dist }}$ & $\sigma_{\text {dist }}$ & $\mu_{\text {card }}$ & $\sigma_{\text {card }}$ \\
\hline \multirow{3}{*}{ 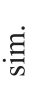 } & 50 & 9.5838 & 0.0952 & 125.9500 & 11.9083 \\
\hline & 100 & 8.3589 & 0.1675 & 88.3900 & 11.4716 \\
\hline & 200 & 6.2449 & 0.2887 & 45.3900 & 9.7210 \\
\hline \multirow{3}{*}{ 『ี } & 50 & 9.8380 & 0.0496 & 156.3400 & 10.1698 \\
\hline & 100 & 9.2845 & 0.1096 & 136.3700 & 10.7607 \\
\hline & 200 & 6.3618 & 0.2721 & 44.7100 & 9.8268 \\
\hline
\end{tabular}

Fig. 3. Performance of the detector on simulated images with simulated and real background and initial object intensities. Sample frames for each setting are shown in Fig. 2.

Cell Excellence wide-field microscope using TIRF microscopy. The sample shows human embryonic kidney cells from the 293 cell line whose SNAP25 protein molecules were marked with the red-fluorescent protein PA-mCherry; furthermore, fiducial markers were added by using cover slips whose coating contains gold beads which are visible at all times. Images were captured on an EMCCD camera chip of size 512 px by 512 px. The field of view represents $54.272 \mu \mathrm{m}$ where the width of each pixel represents $106 \mathrm{~nm}$. The exposure time is $60 \mathrm{~ms}$ which equates to a $16.6 \mathrm{~Hz}$ sampling rate.

To have exact ground truth for the sensor motion available, 20 different linear and Brownian drift trajectories were simulated under the assumption that there is no drift in the video sequence. Here, the velocities of the linear drift were randomly allocated according to a normal distribution with standard deviation 0.25 $\mathrm{px} /$ frame and the process noise had a standard deviation of 0.2

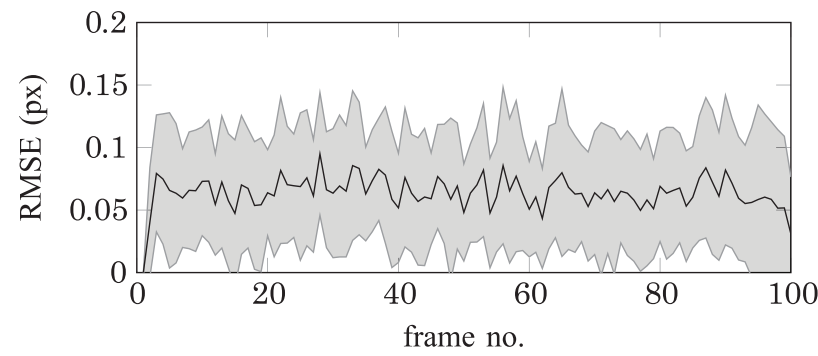

Fig. 4. Average drift in the real data based on 10 fiducial marker positions spread over the whole frame (see Fig. 5(b)) over time. The figure shows the mean RMSE and the standard deviation. Note that $1 \mathrm{px}$ corresponds to $106 \mathrm{~nm}$, so the average RMSE is about $6 \mathrm{~nm}$.

px/frame. The standard deviation of the Brownian motion, on the other hand, was set to $1.0 \mathrm{px} /$ frame.

To ensure that there is no additional drift in the original data, an HISP tracker (see [41]) was used to obtain the trajectories of a manual selection of 10 beads in different regions of the image; those trajectories were moved to the origin and the positions were averaged per frame. In Fig. 4, the RMSE of the averaged bead position to the origin is plotted over all frames and it can be seen that the error stays below $0.1 \mathrm{px}$ which can be expected due to imperfections of the detector.

In order to have a direct comparison between marker-aided and marker-free data, two subframes (see Fig. 5(c)) were chosen where frame (i) is of size $90 \mathrm{px} \times 75 \mathrm{px}$ containing 4 beads and frame (ii) is marker-free and of size $100 \mathrm{px} \times 90 \mathrm{px}$. The average number of objects present per frame is 9.16 in frame (i) and 11.5 in frame (ii). The simulated drifts were imposed on the measurements falling in the respective subframe.

Using PALM, each fluorophore is active exactly once for a short amount of time, furthermore only a fraction of all present molecules emit light simultaneously. This makes it possible to count and localise a huge amount of SNAP25 molecules over time which would highly overlap and thus not be distinguishable if they were active all at once. However, the short life time of objects can make the estimation of the sensor motion challenging, especially if the data contains no fiducial markers. State-of-the-art approaches are image-based and rely on a significant overlap of information between consecutive frames, e.g., in MRI imaging where neighbouring slices are usually very similar. PALM data, on the contrary, is highly changeable and a collection of point-like features of the size of only a few pixels might not be enough to ensure the success of the algorithm. For evaluation purposes, the proposed method was compared with the performance of an image-based registration method, known as StackReg, which is commonly used to correct super-resolution image sequences [42]. StackReg is a plugin for ImageJ that sequentially aligns image frames using an intensity-based least-squares optimisation procedure [43].

The molecules are chosen to be tracked with two motion models in parallel: a Brownian motion model is used to track moving targets, whereas a constant position model is incorporated alongside to take notice of eventual static features in the image. The dynamics noise for the Brownian molecule motion was set to $1 \mathrm{px} /$ frame, furthermore the detection and survival probabilities were chosen to be $P_{D}=0.8$ and $P_{S}=0.99$. The measurement noise was chosen to be $2.0 \mathrm{px} /$ frame in the presence of beads in each image dimension and to $1.5 \mathrm{px} /$ frame in 


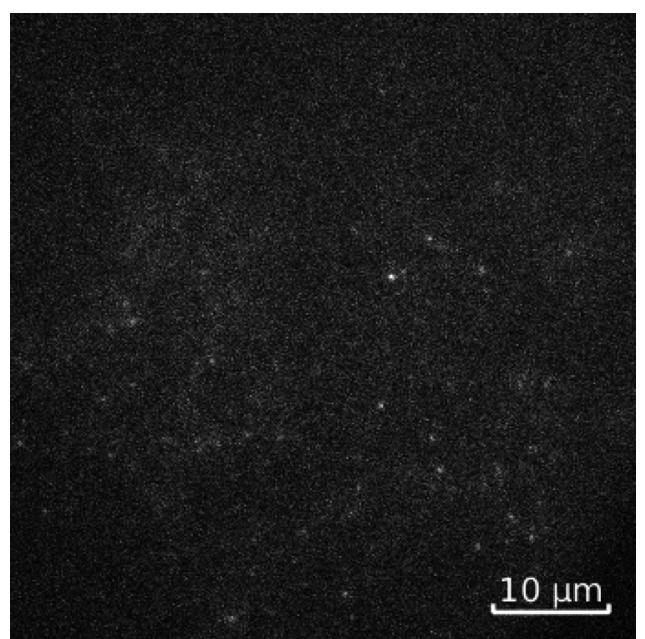

(a)

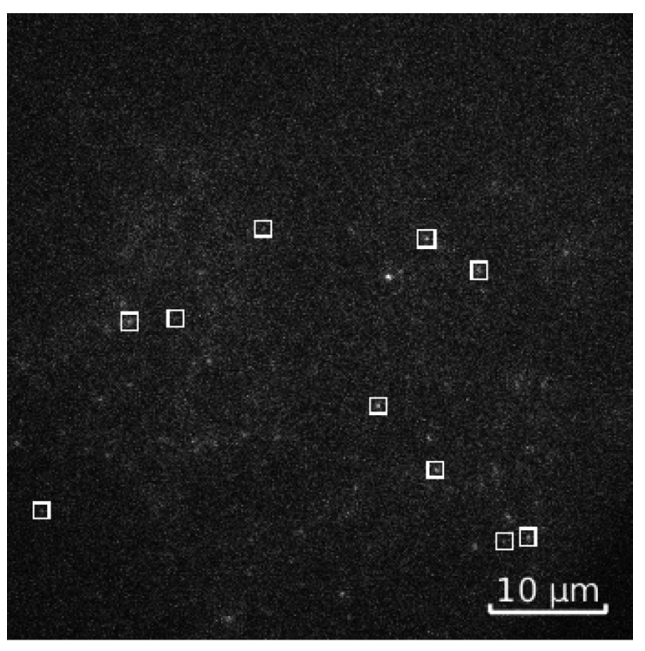

(b)

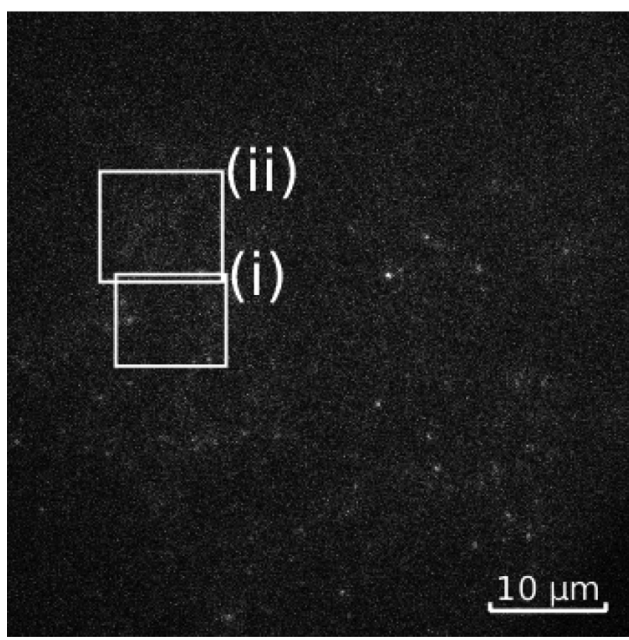

(c)

Fig. 5. Example frame of PALM data used for the drift estimation. (a) Original image. (b) A selection of beads used for the analysis in Fig. 4. Note the difference in intensity due to the decay of the evanescent field of the exiting laser beam. (c) Chosen sub-images: (i) contains at least 4 beads, (ii) marker-free.

the marker-free case; the latter case requires a smaller value to assist the sensor state estimation.

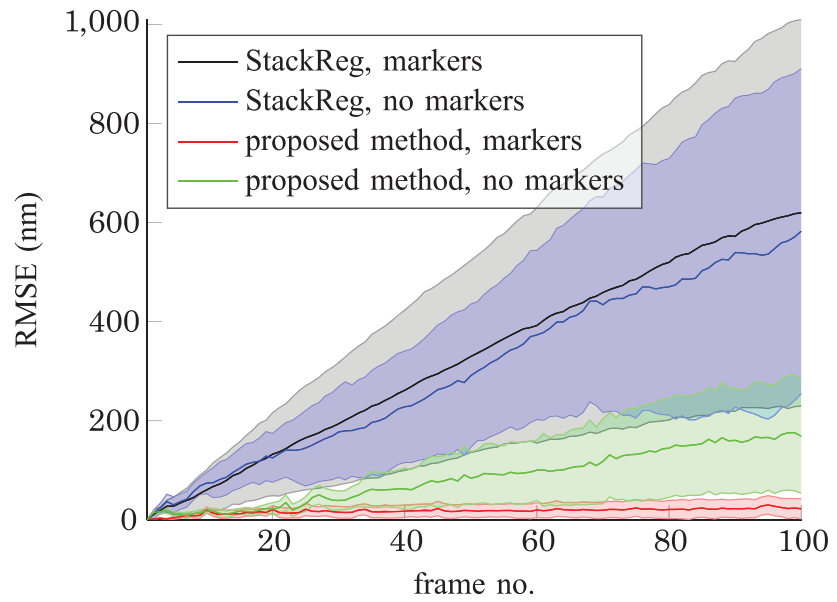

(a)

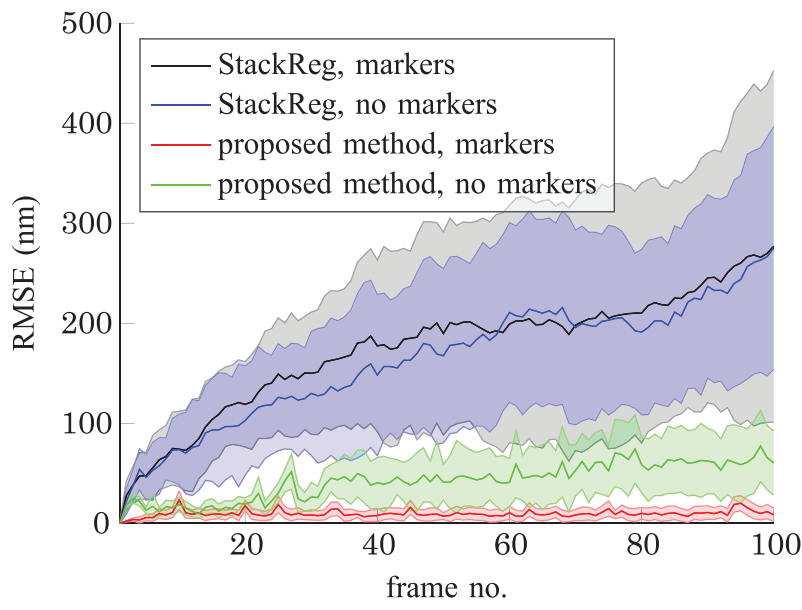

(b)

Fig. 6. Results of the sensor drift estimation the two subimages of $5 \mathrm{c}$ for Brownian and linear motion, all plotted with their standard deviation over all MC runs. (a) Estimation of linear drift. (b) Estimation of Brownian drift.

On the other hand, the particle filter was executed under the assumption of a Brownian motion model where 200 particles were used throughout all experiments. In the case of linear motion the process noise was set to $0.5 \mathrm{px} /$ frame and for the Brownian case to $1.0 \mathrm{px} /$ frame. For the multi-object likelihood (14), only the static objects were considered since they are more reliable. Note here that not only the markers are static in the present example, but also many of the appearing targets.

To compensate for the randomness of particle filtering, the results for each trajectory were averaged over 50 Monte Carlo runs. Moreover, 20 different trajectories for both Brownian and linear drift were analysed to exclude singularities in performance. Fig. 6 shows the averaged estimation results for image-based and probabilistic registration on both subimages shown in Fig. 5(c) in terms of the Root Mean Square Error (RMSE). In both cases, the image-based approach performs similarly on the marker-aided (black) and marker-less (blue) subframes, where the random motion is estimated slightly better than the linear motion. Also, the displayed standard deviation shows that the estimation uncertainty increases significantly over time. The proposed method, on the other hand, outperforms the image-based registration by a factor 5 in the marker-free case (green) and it even recovers the drift with 
an RMSE of less than $10 \mathrm{~nm}$, i.e., $0.1 \mathrm{px}$ over 100 time steps in presence of fiducial markers (red). A video of the results is available as supplementary material.

The complexity depends linearly on the number of particles, furthermore it can be easily parallelised since the PHD filters are processed independently. A runtime analysis of a GPU implementation of the single-cluster PHD filter can be found in [44].

\section{CONCLUSIONS}

In this work, a PHD filter with classification was combined with a particle filter inspired by simultaneous localisation and mapping (SLAM) to provide an intertwined estimation of the sensor movement with respect to the monitored sample and the intracellular motion of molecular structures in photo-activated localisation microscopy (PALM). This type of filter allows to distinguish objects with different motion behaviours which can be exploited in the calibration. Being an important aspect of the performance of tracking algorithms, the chosen detection method was evaluated on simulated data to demonstrate its performance under different conditions. On the other hand, the joint sensor and object state estimation was tested on real data with artificial sensor drift. Here, two subimages of the data set were compared where one contained fiducial makers and the other was marker-free. A comparison with an existing image-based method showed that the proposed algorithm outperforms commonly used state-of-the-art techniques and that it is able to estimate sensor drift even in data sets where no fiducial markers are available.

\section{ACKNOWLEDGMENT}

The authors would like to thank Francesco Tonolini, Isabella McKenna and Rachael Tobin (SUPA, Heriot-Watt University, Edinburgh) for their support and their work on object detection.

\section{REFERENCES}

[1] D. J. Stephens and V. J. Allan, "Light microscopy techniques for live cell imaging," Sci., vol. 300, no. 5616, pp. 82-86, 2003.

[2] E. Betzig, G. H. Patterson, R. Sougrat, O. W. Lindwasser, S. Olenych, J. S. Bonifacino, M. W. Davidson, J. Lippincott-Schwartz, and H. F. Hess, "Imaging intracellular fluorescent proteins at nanometer resolution," Sci., vol. 313, no. 5793, pp. 1642-1645, 2006.

[3] S. T. Hess, T. P. Girirajan, and M. D. Mason, "Ultra-high resolution imaging by fluorescence photoactivation localization microscopy," Biophysical J., vol. 91, no. 11, pp. 4258-4272, 2006.

[4] J. Lippincott-Schwartz and G. H. Patterson, "Development and use of fluorescent protein markers in living cells," Sci., vol. 300, no. 5616, pp. 87-91, 2003.

[5] N. L. Thompson, T. P. Burghardt, and D. Axelrod, "Measuring surface dynamics of biomolecules by total internal reflection fluorescence with photobleaching recovery or correlation spectroscopy," Biophysical J., vol. 33, no. 3, pp. 435-454, 1981.

[6] S. Manley, J. M. Gillette, and J. Lippincott-Schwartz, "Chapter five-single-particle tracking photoactivated localization microscopy for mapping single-molecule dynamics," in Single Molecule Tools, Part B:Super-Resolution, Particle Tracking, Multiparameter, and Force Based Methods, ser. Methods in Enzymology, N. G. Walter, Ed. New York, NY, USA: Academic, 2010, vol. 475, pp. 109-120.

[7] I. Smal, E. Meijering, K. Draegestein, N. Galjart, I. Grigoriev, A. Akhmanova, M. van Royen, A. Houtsmuller, and W. Niessen, "Multiple object tracking in molecular bioimaging by Rao-Blackwellized marginal particle filtering," Med. Image Anal. Special Iss. Inf. Process. Med. Imag., vol. 12, no. 6, pp. 764-777, 2008.

[8] D. Sage, H. Kirshner, T. Pengo, N. Stuurman, J. Min, S. Manley, and M. Unser, "Quantitative evaluation of software packages for single-molecule localization microscopy," Nature Meth., vol. 12, pp. 717-724, 2015.
[9] S. H. Lee, M. Baday, M. Tjioe, P. D. Simonson, R. Zhang, E. Cai, and P. R. Selvin, "Using fixed fiduciary markers for stage drift correction," Opt. Exp., vol. 20, no. 11, pp. 12177-12183, May 2012.

[10] R. Brunelli, Template Matching Techniques in Computer Vision: Theory and Practice. New York, NY, USA: Wiley, 2009.

[11] C. Geisler, T. Hotz, A. Schönle, S. W. Hell, A. Munk, and A. Egner, "Drift estimation for single marker switching based imaging schemes," Opt. Exp., vol. 20, no. 7, pp. 7274-7289, Mar. 2012.

[12] K. Miura, "Tracking movement in cell biology," in Microscopy Techniques, ser. Advances in Biochemical Engineering, J. Rietdorf, Ed. Berlin, Heidelberg, Germany: Springer, 2005, vol. 95, pp. 267-295.

[13] Y. Kalaidzidis, "Multiple objects tracking in fluorescence microscopy," J. Math. Biol., vol. 58, no. 1-2, pp. 57-80, 2009.

[14] Y. Bar-Shalom and T. E. Fortmann, Tracking and Data Association. San Diego, CA, USA: Academic, 1987.

[15] W. Godinez, M. Lampe, S. Wörz, B. Müller, R. Eils, and K. Rohr, "Deterministic and probabilistic approaches for tracking virus particles in time-lapse fluorescence microscopy image sequences," Med. Image Anal. Includes Special Sec. Function. Imag. Model. of the Heart, vol. 13, no. 2, pp. 325-342, 2009.

[16] I. Smal, W. Niessen, and E. Meijering, "A new detection scheme for multiple object tracking in fluorescence microscopy by joint probabilistic data association filtering," in Proc. 5th IEEE Int. Symp. Biomed. Imag.: From Nano to Macro (ISBI'08), May 2008, pp. 264-267.

[17] S. Rezatofighi, S. Gould, R. Hartley, K. Mele, and W. Hughes, "Application of the IMM-JPDA filter to multiple target tracking in total internal reflection fluorescence microscopy images," in Medical Image Computing and Computer-Assisted Intervention-MICCAI 2012, ser. Lecture Notes in Computer Science, N. Ayache, H. Delingette, P. Golland, and K. Mori, Eds. Berlin-Heidelberg, Germany: Springer, 2012, vol. 7510, pp. 357-364.

[18] N. Chenouard, I. Bloch, and J. Olivo-Marin, "Multiple hypothesis tracking for cluttered biological image sequences," IEEE Trans. Pattern Anal. Mach. Intell., vol. 35, no. 11, pp. 2736-3750, Nov. 2013.

[19] R. P. Mahler, "Multitarget Bayes filtering via first-order multitarget moments," IEEE Trans. Aerosp. Electron. Syst., vol. 39, no. 4, pp. 1152-1178, Oct. 2003.

[20] R. P. Mahler, Statistical Multisource-Multitarget Information Fusion. Boston, MA, USA: Artech House, 2007.

[21] L. Lin, Y. Bar-Shalom, and T. Kirubarajan, "Track labeling and PHD filter for multitarget tracking," IEEE Trans. Aerosp. Electron. Syst., vol. 42, no. 3, pp. 778-795, Jul. 2006.

[22] T. Li, S. Sun, J. Corchado, and M. F. Siyau, "A particle dyeing approach for track continuity for the SMC-PHD filter," in Proc. 17th Int. Conf. Inf. Fusion (FUSION), Jul. 2014, pp. 1-8.

[23] B.-T. Vo, B.-N. Vo, and A. Cantoni, "Analytic implementations of the cardinalized probability hypothesis density filter," IEEE Trans. Signal Process., vol. 55, no. 7, pp. 3553-3567, Jul. 2007.

[24] A. Swain and D. Clark, "The single-group PHD filter: An analytic solution," in Proc. 14th Int. Conf. Inf. Fusion (FUSION), 2011, pp. 1-8.

[25] B. Ristic, D. Clark, and N. Gordon, "Calibration of multi-target tracking algorithms using non-cooperative targets," IEEE J. Sel. Topics Signal Process., vol. 7, no. 3, pp. 390-398, Jun. 2013.

[26] C. Lee, D. Clark, and J. Salvi, "SLAM with dynamic targets via singlecluster PHD filtering," IEEE J. Sel. Topics Signal Process., vol. 7, no. 3, pp. 543-552, Jun. 2013.

[27] J. Houssineau, D. Clark, S. Ivecovic, C. S. Lee, and J. Franco, “A unified approach for multi-object triangulation, tracking and camera calibration," ArXiv E-prints, 2014.

[28] I. Schlangen, J. Houssineau, and D. Clark, "A novel approach to image calibration in super-resolution microscopy," in Proc. Int. Conf. Control, Autom., Inf. Sci. (ICCAIS), Dec. 2014, pp. 111-116.

[29] J. Franco, J. Houssineau, D. E. Clark, and C. Rickman, "Simultaneous tracking of multiple particles and sensor position estimation in fluorescence microscopy images," in Proc. 2nd Int. Conf. Control, Autom., Inf. Sci. (ICCAIS), 2013.

[30] E. Betzig, G. H. Patterson, R. Sougrat, O. W. Lindwasser, S. Olenych, J. S. Bonifacino, M. W. Davidson, J. Lippincott-Schwartz, and H. F. Hess, "Imaging intracellular fluorescent proteins at nanometer resolution," Sci., vol. 313, pp. 1642-1645, Sep. 2006.

[31] I. Smal, M. Loog, W. Niessen, and E. Meijering, "Quantitative comparison of spot detection methods in fluorescence microscopy," IEEE Trans. Med. Imag., vol. 29, no. 2, pp. 282-301, Feb. 2010.

[32] B. Zhang, J. Zerubia, and J.-C. Olivo-Marin, "Gaussian approximations of fluorescence microscope point-spread function models," Appl. Opt., vol. 46, no. 10, pp. 1819-1829, 2007. 
[33] S. Rezatofighi, S. Gould, B.-N Vo, K. Mele, W. Hughes, and R. Hartley, "A multiple model probability hypothesis density tracker for time-lapse cell microscopy sequences," in Inf. Process. Med. Imag., ser. Lecture Notes in Computer Science, J. Gee, S. Joshi, K. Pohl, W. Wells, and L. Zöllei, Eds. Berlin, Heidelberg, Germany: Springer, 2013, vol. 7917, pp. 110-122.

[34] J. Houssineau and D. Laneuville, "PHD filter with diffuse spatial prior on the birth process with applications to GM-PHD filter," in Proc. 13th Conf. Inf. Fusion (FUSION), 2010, pp. 1-8.

[35] B. Ristic, D. Clark, and B.-N. Vo, "Improved SMC implementation of the PHD filter," in Proc. 13th Conf. Inf. Fusion (FUSION), 2010, pp. $1-8$.

[36] B.-N. Vo and W.-K. Ma, "The Gaussian mixture probability hypothesis density filter," IEEE Trans. Signal Process., vol. 54, no. 11, pp. 4091-4104, Nov. 2006.

[37] K. Panta, D. Clark, and B.-N. Vo, "Data association and track management for the Gaussian mixture probability hypothesis density filter," IEEE Trans. Aerosp. Electron. Syst., vol. 45, no. 3, pp. 1003-1016, Jul. 2009.

[38] A. Doucet, N. de Freitas, and N. Gordon, Sequential Monte Carlo Methods in Practice. Berlin, Germany: Springer-Verlag, 2001, vol. 1.

[39] S. H. Rezatofighi, W. T. E. Pitkeathly, S. Gould, R. Hartley, K. Mele, W. E. Hughes, and J. G. Burchfield, "A framework for generating realistic synthetic sequences of total internal reflection fluorescence microscopy images," in Proc. IEEE 10th Int. Symp. Biomed. Imag.: From Nano to Macro, San Francisco, CA, USA, 2013.

[40] D. Schuhmacher, B.-T. Vo, and B.-N. Vo, "A consistent metric for performance evaluation of multi-object filters," IEEE Trans. Signal Process., vol. 56, no. 8, pp. 3447-3457, Aug. 2008.

[41] J. Houssineau, P. Del Moral, and D. E. Clark, "General multi-object filtering and association measure," in Proc. 5th IEEE Int. Workshop Comput. Adv. Multi-Sensor Adaptive Process. (CAMSAP), 2013, pp. $33-36$.

[42] D. Lim, "Achieving accurate image registration as the basis for superresolution," M.S. thesis, Univ. of Western Australia, Crawley, WA, Australia, 2003.

[43] P. Thévenaz, U. Ruttimann, and M. Unser, "A pyramid approach to subpixel registration based on intensity," IEEE Trans. Image Process., vol. 7, no. 1, pp. 27-41, Jan. 1998.

[44] C. S. Lee, J. Franco, J. Houssineau, and D. Clark, "Accelerating the single cluster PHD filter with a GPU implementation," in Proc. Int Conf. Control, Autom. Inf.. Sci. (ICCAIS), Dec. 2014, pp. 53-58.

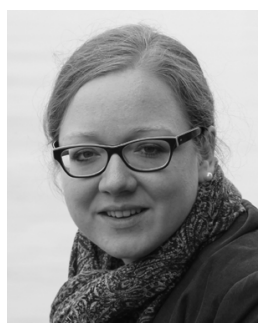

Isabel Schlangen is a current Ph.D. studentship holder at the Edinburgh Super-Resolution Imaging Consortium, UK. She received a German diploma in mathematics from the University of Bonn (Germany) in 2012 and a joint M.Sc. degree in vision and robotics from the Universities of Burgundy (France), Girona (Spain), and Heriot-Watt (Edinburgh, UK) in 2014. Her current research interests are multi-target estimation, probability theory and image analysis in a mainly biomedical context.

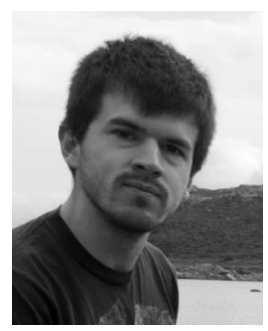

José Franco received a B.Sc. degree in mathematical engineering from EAFIT University in 2010 and a M.Sc. in computer vision and robotics jointly from the University of Burgundy, the University of Girona and Heriot-Watt University in 2013. He is currently a doctoral student in Heriot Watt University under the supervision of Dr. Daniel Clark, where he studies multiple object estimation techniques for a variety of applications.

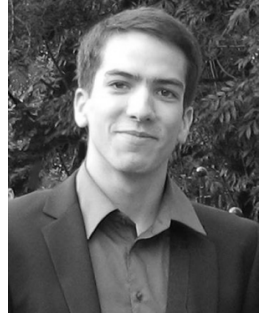

Jérémie Houssineau received an Eng. degree in mathematical and mechanical modelling from MATMECA, Bordeaux, and a M.Sc. degree in mathematical modelling and statistics from the University of Bordeaux, both in 2009. From 2009 to 2011, he was a Research Engineer with DCNS, Toulon, and then with INRIA Bordeaux. He received his $\mathrm{Ph} . \mathrm{D}$. degree in statistical signal processing from Heriot-Watt University, Edinburgh, in 2015. His research interests include applied probability, point process theory and multi-object estimation.

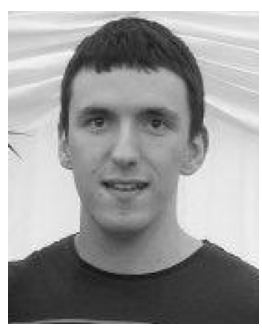

William T. E. Pitkeathly is a Research Associate at Heriot-Watt University, Edinburgh, UK, in the biological chemistry, biophysics and bioengineering working on super-resolved three dimensional imaging. In 2013, he completed a 4-year MSc/PhD course from the Physical Sciences of Imaging in the Bio-medical Sciences doctoral training center, University of Birmingham, UK, following his B.Sc. in physics at the same university. His current research interests include: development of new multi-dimensional fluorescence microscopy techniques, bio-medical imaging, and fluorescence microscopy image analysis.

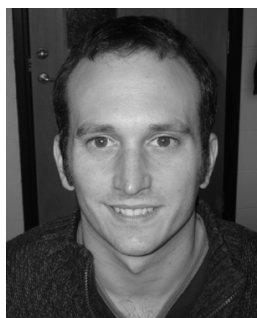

Daniel Clark is an Associate Professor in the School of Engineering and Physical Sciences at Heriot-Watt University. His research interests are in the development of the theory and applications of multi-object estimation algorithms for sensor fusion problems. He has led a range of projects spanning theoretical algorithm development to practical deployment. He was awarded his Ph.D. in 2006 from Heriot-Watt University.

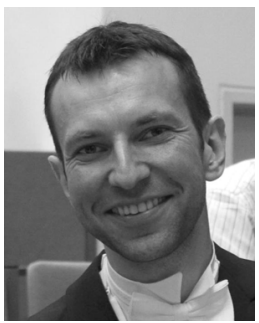

Ihor Smal received a M.Sc. degree (summa cum laude) in electrical engineering from Ivan Franko National University of Lviv, Ukraine, in 1999, and a Professional Doctorate in Engineering (PDEng) degree from Eindhoven University of Technology, the Netherlands, in 2005. In 2009 he defended his Ph.D. thesis on particle filtering methods for bioimaging a the Erasmus University Rotterdam, the Netherlands. In February 2009, he joined the Biomedical Imaging Group Rotterdam of the Erasmus MC-University Medical Center Rotterdam, as a Postdoc. His current research focuses on cellular and molecular image and data analysis using Bayesian statistics, sequential and Markov Chain Monte Carlo methods.

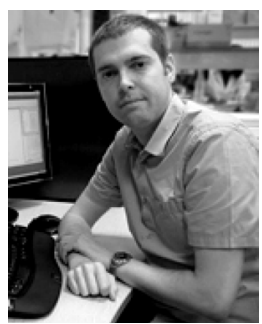

Colin Rickman has worked in the field of membrane trafficking and fusion for the last ten years. Over this period he has published a number of research papers in high ranking journals on the protein interactions underlying regulated membrane fusion in endocrine and neuronal regulated fusion. During his doctoral training and subsequent postdoctoral position (MRC Laboratory of Molecular Biology, Cambridge) he applied a broad range of protein biochemistry techniques to describe a cascade of protein-protein interactions regulating multiple steps of the fusion process. As an Associate Professor in the Life-Physical Sciences Interface at Heriot-Watt University he has established key collaborations with physicists and mathematicians to provide inventive routes for data acquisition and analysis. 